\title{
Does Competence Determine Who Leads in a Dyadic Cooperative Task? A Study of Children with and without a Neurodevelopmental Disorder
}

\author{
Roy Vink (D), Fred Hasselman $(\mathbb{D}$, Antonius H. N. Cillessen $(\mathbb{D}$, \\ Maarten L. Wijnants (D), and Anna M. T. Bosman iD \\ Behavioural Science Institute, Radboud University, Netherlands \\ Correspondence should be addressed to Roy Vink; r.vink@pwo.ru.nl
}

Received 4 May 2018; Revised 15 August 2018; Accepted 30 August 2018; Published 1 November 2018

Academic Editor: Ruud den Hartigh

Copyright (C) 2018 Roy Vink et al. This is an open access article distributed under the Creative Commons Attribution License, which permits unrestricted use, distribution, and reproduction in any medium, provided the original work is properly cited.

Cooperative learning is an effective means for the acquisition of academic performance. It is an established fact that collaborating members should be operating in one another's zone of proximal development to attain optimal performance. One variable that plays an as-yet unknown role in collaborative success is the leader-follower distinction. In the present study, leading and following behavior was determined by assessing rhythmical coordination of postural sway in typically developing children $(n=183)$ and children with a neurodevelopmental disorder $(n=106)$. Postural sway was measured using Nintendo Wii Balance Boards, and dyads performed a tangram task while standing on these balance boards, with the number of puzzles solved correctly serving as the measure of task performance. Irrespective of task performance, there was a consistent pattern of leading and following in typically developing dyads: the higher-ability child was in the lead. For children with a neurodevelopmental disorder, the pattern differed depending on task performance. While the patterns of low-performing dyads were comparable to those of typically developing children, high-performing dyads showed the opposite pattern; namely, the low-ability dyad member was in the lead. For interactions with children with a neurodevelopmental disorder and a low-level cognitive ability, it may be better to follow their lead, because it may result in better performance on their part.

\section{Introduction}

Cooperative learning refers to a method in which two or more individuals work together in small groups towards a common goal with the aim of helping one another in the acquisition of academic knowledge [1]. Its success is to a large extent affected by positive interdependence, individual accountability, positive interactions, appropriate social skills, and group processing (for a more detailed description, see [2]). Cooperative learning appears to be rather effective. Johnson, Maruyama, Johnson, Nelson, and Skon [3] reviewed 122 studies on the effects of cooperative, competitive, and individualistic goal structures and showed that cooperative groups performed better than competitive groups and individuals. More recently, Roseth, Johnson, and Johnson [4] conducted a similar meta-analysis, examining nearly 150 studies. They also found that cooperative goal structures were related to better task performance than competitive or individual goal structures.

An important factor that affects the outcome of cooperation is dyad composition. Vygotsky [5], for example, stated that the ability level of the cooperating individuals is crucial and that the determining factor for successful cooperation is that one individual is, or moves within, the other's zone of proximal development (ZPD). Vygotsky defined the ZPD as "the distance between the actual developmental level as determined by independent problem solving and the level of potential development as determined through problem solving under adult guidance or in collaboration with more capable peers" (p. 86). Vygotsky believed that the ZPD is important for cognitive growth. A child who is learning within his or her ZPD can do things with the help of a more experienced person (peer or adult) that he or she was not (yet) able to do alone. Thus, for cooperative learning 
to be successful, from a Vygotskian point of view, there has to be active interaction (explaining and reasoning) between individuals who differ in levels of expertise. This in turn may result in intersubjectivity or a shared understanding through discussion of different viewpoints [6,7]. Although cooperative learning has been shown to be successful, working in Vygotsky's zone of proximal development is not the only reason for that [8]. Task performance is not just determined by cognitive ability level, but also by other factors as well. One of these factors is who takes the lead and who follows the lead of the interaction partner.

1.1. Leading-Following Behavior. Being a leader or a follower has been related to academic achievement and peer evaluations. Dingel and Wei [9] studied introductory sociology students who participated in an interdisciplinary project. In this project, they collaboratively wrote three papers in groups of four to six students. In the final week of class, students were presented with a survey, in which they were asked to indicate, among other things, whether they felt like a leader and a follower in their own group (both could be answered with yes or no). Dingel and Wei found that not only leaders had higher peer evaluations than followers, but also they received higher average grades than followers. Dunbar, Dingel, Dame, Winchip, and Petzold [10] found similar results; leaders had higher grades and higher social self-efficacy than followers.

Notwithstanding the significance of these findings, they examined leader-follower behavior at a macro level, that is, as an outcome measure. An alternative perspective is studying the behavior at a micro level by looking at the process (or dynamics) that underlies macro-level observable behavior. Wichers' [11] study provides a recent example in which micro-level patterns revealed the development of symptoms of depression. In the present study, we studied the microlevel dynamics of postural sway to provide insight into the working mechanisms that underlie leader-follower behavior in a dyadic cooperative task.

During a task in which two people have to work together to perform it, bodily movements have to be adjusted to one another. An unobtrusive measure is postural sway; it reveals the (un)conscious back-and-forward as well as leftto-right movements of an individual. The movements of one individual can entrain those of the cooperative partner in the dyad. The one who initiates the movement is called the leader and the one who follows the follower. Because all tasks require behavior and all behavior requires movement, postural sway appears to be an excellent exemplar variable to investigate the underlying pattern of cooperation and leaderfollower behavior (e.g., [12]). More concretely, we aim at investigating whether leader-follower behavior is related to task performance over the course of an interaction (i.e., across time), but also whether similar or different mechanisms are at work in different populations (i.e., typically developing children and children with a neurodevelopmental disorder).

One way to analyze these micro processes over time is by means of Cross Recurrence Quantification Analysis (explained in more detail in Methods section). Guevara, Cox, van Dijk, and van Geert [13] have shown its potential for studying cooperative behavior, Leonardi, Nomikou, Rohlfing, and Rączaszek-Leonardi [14] for leader-follower behavior in mother-child interaction, and Warlaumont, Richards, Gilkerson, and Oller [15] for leader-follower behavior in typically developing infants and infants with ASD. In the next paragraph, we explain leading-following behavior in terms of rhythmical coordination.

1.2. Rhythmical Coordination. Leading and following behavior of a dyad has been studied during rhythmical coordination of bodily movements and the outcome of a cooperative interaction is related to the level of interpersonal synchrony or interpersonal coordination (e.g., [16-18]). Synchrony involves engaging in the same action (i.e., the spatial aspect) at (about) the same time (i.e., the temporal aspect). For example, when two people are walking side by side and their stride-intervals are the same, their behavior is synchronized. Coordination is about timing, the when (i.e., the temporal aspect), and not so much about what is being done, the what (i.e., the spatial aspect). For example, when two individuals are lifting a table together, it does not matter how either one of them does it, as long as they lift it at the same time. Here we focus on interpersonal coordination, that is, on the timing or rhythm of behavior.

Jaffe et al. [19] defined rhythm as "... a recurrent nonrandom patterning that may or may not be strictly regular" (p. 1). All motor and vocal behavior has a rhythm [20]. This rhythm reveals information about the interaction partner [21]. Coordination of (vocal) rhythms always takes place in relation to those of the interaction partner and, interestingly, the timing of preverbal dialogues appears similar to that of verbal dialogues in adults [22]. Infants are already equipped with the tools for conversation before they can talk. It is through expectancies and anticipation, knowing what the other will do in relation to what you are doing, that this kind of coordination of timing patterns (e.g., when to pause, or whose turn it is) is possible [22]. Synchronized behavior generally leads to positive effect, whereas individual rhythms that are not properly coordinated can cause a feeling of uneasiness (e.g., [23, 24]).

It is still unclear whether it is better to have high or low levels of coordination. Chapple (in [19]) argued that it is better to have high levels of coordination, whereas Gottman [25] stated that high levels of coordination are related to distress in communication. More recent work has shown that in some situations the preferred level of coordination depends on the environment or task demands [16-18], thus challenging the idea that it should be either high or low. Vink et al. [17] asked 183 dyads of primary-school children to perform a tangram task while standing on a Nintendo Wii Balance Board. The balance board recorded each child's postural sway. The results showed that task performance was better when the dyad's postural sway was loosely synchronized. Dyads that performed better had less deterministic postural sway patterns than dyads that performed worse. However, this was only the case for postural sway movement on the $\mathrm{x}$ axis. According to the authors, this indicated that better task performance demanded more coordination rather than synchronization. That is, the less deterministic postural sway patterns indicated that the periods of synchronized dyadic 
postural sway were shorter in the better performing dyads, which may suggest that it is more important to coordinate than synchronize.

In a follow-up study, Vink et al. [18] analyzed the displacement of postural sway, instead of the separate $\mathrm{x}$ and y-axis measurements used by Vink et al. [17], and they examined a different outcome measure, the level of entropy of the dyadic postural sway. Vink et al. showed that in better performing dyads the level of entropy was lower than in worse performing dyads, indicating that there was more order in better performing dyads' postural sway patterns. Combining this with the results of their previous study, Vink et al. again concluded that coordination is sometimes more important than synchronization, since better performance was indicated by more ordered, shorter periods of similar postural sway, and dyads continuously adjust their postural sway to that of their interaction partner.

Abney et al. [16] also maintained that in certain interactions it is better when dyads are more loosely coupled. They asked participants to perform a dyadic problem-solving task in which they had to create an as high as possible tower from raw spaghetti and marshmallows. One participant had control over the spaghetti, while the other handled the marshmallows. When dyads were more loosely coupled, that is, more coordinated, their performance was better. In addition, Abney et al. showed that performance also depended on the role division within each dyad. Although their results did not reach significance, they did point to the possibility that "... the emergence of role-sensitive temporal organization may be vital to effective performance in highly constrained dyadic problem solving" (p. 321).

Not everyone, however, is able to rhythmically coordinate smoothly. Individuals who suffer from a neurodevelopmental disorder, such as people with autism, have been shown to not only experience difficulties communicating [26], but, almost all of them, suffer from motor control problems, which in turn may add to synchronization problems (Pettersson, Anckarsäter, Gillberg \& Lichtenstein, 2013). Tiegerman and Primavera showed that communicating was hampered to a large extent by gaze aversion (i.e., not wanting to look at other people's faces). Interestingly, imitating or synchronizing the behavior of the individual with a neurodevelopmental disorder may enhance communication; when the experimenter imitated the actions of the autistic child, there was an increase in gaze frequency and gaze duration, as opposed to when the experimenter did not imitate the autistic child's behavior.

Additional support for this idea came from Trevarthen and Daniel [27]. They observed different rhythms in the interactions between a father and his monozygotic twin daughters. At the age of two, one of the girls was diagnosed with autism. The videos that were analyzed were made when the girls were 11 months old, long before one of them was diagnosed with autism. Trevarthen and Daniel's analysis revealed that the father interacted differently with his two daughters. In the interaction with his nonautistic child a clear rhythm (i.e., coherent temporal regulation) was visible, whereas in the interactions with the autistic child this rhythm was absent. Moreover, to encourage his autistic daughter to engage in the interaction, he took the lead, whereas the interactions with his nonautistic daughter revealed more following behavior. Although this behavior feels natural to most of us, Gernsbacher [28] suggested a counter intuitive notion, namely, that children with a neurodevelopmental disorder may actually need to be in the lead and have a more capable interaction partner to follow their lead. This idea adds to Vygotsky's [5] theory, in that the more skilled individual has to position him- or herself within the less skilled individual's zone of proximal development and from there the more skilled individual should follow the lead of the less skilled individual.

Vink et al. [18] examined whether typically developing children and children with a neurodevelopmental disorder (e.g., autism and ADHD) differed in task performance and coordination of postural sway. The children had to cooperate in solving tangram puzzles, while their postural sway was recorded. As expected, the results showed that children with a neurodevelopmental disorder performed significantly worse on the tangram task than their typically developing peers. However, when studying their postural sway during this cooperative process, the entropy effect was the same in both groups: lower levels of entropy (i.e., reduced disorder of synchrony) were related to better task performance. In other words, dyads performed better when their postural sway was more coordinated. This suggests that the nature of the interaction is more important than the disorder to explain the communication difficulties. In the Trevarthen and Daniel [27] study, the problem may not have been that one child was autistic and the other was not, but a mismatch between the natural rhythms of the interaction partners in case of the interaction between the father and the autistic child. These findings led us to wonder what could account for observed differences in the outcome of a cooperative task, if it is not the level of coordination. Could it be different patterns of leading and following?

1.3. Present Study. This study addresses three questions. One, who leads and who follows in a cooperative task? Previous research has shown that more skilled people are often leaders and Hooper [8] showed that homogeneous high-ability dyads had superior performance, whereas average-ability homogeneous dyads were the poorest performers on a cooperative task. However, and related to the second question, how roles are divided may depend on task performance. Two, are leader-follower patterns related to task performance? A conjecture is that in better performing dyads the more skilled child is the follower, whereas in worse performing dyads the more skilled child is the leader. Three, is there a difference in leader-follower patterns between typically developing children and children with a neurodevelopmental disorder? As Leonardi et al. [14] showed, typically developing children may not be in need of follower over the course of their development, whereas children with a neurodevelopmental disorder may profit from a more skilled follower. We will therefore investigate whether leader-follower behavior differs between typically developing children and children with a neurodevelopmental disorder when they cooperate on a cognitive task. 


\section{Method}

2.1. Participants. Children were randomly assigned to a same-sex dyad, because only same-sex (not mixed-sex) dyads perform better together than they do individually [29]. Not all dyads that participated were included in the study. Reasons for exclusion were either technical failures with data recording or an uneven number of children in a classroom, which led to one child participating in two dyads or in a dyad that was not same-sex. The group of typically developing children consisted of 183 dyads attending regular-primary education $\left(M_{\mathrm{age}}=10 ; 8\right.$ years, $S D=1 ; 00$, range: 8-13, 95 boys and 88 girls). The group of atypically developing children consisted of 106 dyads attending special-primary education $\left(M_{\text {age }}=10 ; 10, S D=1 ; 3\right.$, range: $8-13 ; 74$ boys and 32 girls $)$. Note that, in Netherlands, inclusive education is not yet fully implemented. A large group of children with special needs are referred to special-primary education. They do not necessarily have an official DSM diagnosis (although many of them do), but all of them show behavior that is reminiscent of a developmental disorder. Due to the large diversity within this group, it is difficult to draw conclusions about each of the disorders that are present. Therefore, we chose to look at this group as a group of children with a developmental disorder (i.e., the commonality) and how this group differs from its typically developing counterpart.

Letters were sent to a large number of Dutch regular and special-primary schools to request participation. After two weeks, schools were asked whether or not they wanted to participate. Schools that responded positively received additional information by email, including a letter for the parents in which they were informed about the study and asked for permission for their child's participation. A passive consent procedure was followed.

We did not seek approval from the Ethics Committee for conducting the research related to the research project 'Synchronizing to Learn and Like.' The reason was that, within the Behavioural Science Institute, it was not customary to do so at the time this research was conducted. Only research using invasive methods required approval from the Ethics Committee. The present study was noninvasive and did not pose any threats to the participants in whatever possible way.

\subsection{Materials and Procedure}

2.2.1. Nintendo Wii Balance Boards. Postural sway of both dyad members was recorded using two Nintendo Wii Balance Boards (WBBs; Nintendo, Kyoto, Japan). The WBB is a reliable, easily moveable, and inexpensive alternative to the less portable and more expensive force platforms often used in clinical settings $[30,31]$. A custom-made Windows-based program recorded the two WBBs simultaneously (Voogt, TSG-FSW, Radboud University, The Netherlands). Sampling rate was set at $100 \mathrm{~Hz}$ and the collected data provided information about postural sway in both the medial-lateral (x-axis) and anterior-posterior (y-axis) direction.

2.2.2. Tangram Task. A tangram puzzle consists of seven pieces: two large triangles, one medium triangle, two small triangles, a square, and a rhomboid (see Figure 1). These pieces can be used to create all kinds of figures. The figures that the dyads had to recreate were printed on A4 paper.

The experiment took place at school, in a room in which a table was present. Table height was adjusted to the needs of each of the dyads. The children performed the task both individually and cooperatively. Prior to the individual task, the children were verbally informed by the experimenter who demonstrated how the task was to be performed using an example tangram puzzle. The children had to lay the tangram pieces on top of printed figures on A4-paper. There were three different sets consisting of 18 tangram puzzles each: sets $A$ and $\mathrm{C}$ were used for the individual part, set $\mathrm{B}$ for the cooperative part.

During both the individual and cooperative parts of the task, children had 10 minutes to recreate as many tangram puzzles as possible. The number of puzzles correctly solved when they performed the task individually served as the pretest measure. This pretest measure was used to determine which of the dyad members was the more competent one. The number of puzzles solved correctly when they performed the task together served as the measure for the cooperative task score. During these 10 minutes, they stood on the $\mathrm{WBB}$, and their postural sway was monitored. Children were allowed to move, as long as they did this on the WBB. During the individual part the WBBs were approximately 70 centimeters apart, while during the cooperative part they were approximately 10 centimeters apart. After finishing a puzzle, the researcher checked whether it was correct. If so, the child or dyad was allowed to continue to the next puzzle, otherwise they were asked to keep trying. Only when a child or dyad made many unsuccessful attempts or became very frustrated were they allowed to skip a puzzle. After 10 minutes, children were told to stop and asked to step off of the WBB. The number of correctly recreated puzzles was the task performance score. After finishing the experiment, as a token of gratitude for their participation, the children were given a small present (e.g., a pen or pencil).

\subsection{Data Preparation and Analysis}

2.3.1. Cross Recurrence Quantification Analysis. Data reduction was first performed on the original data, given the computational intensity of the analyses. We down sampled the data to $5 \mathrm{~Hz}$ (the original data was sampled at $100 \mathrm{~Hz}$ ), resulting in time series of approximately 3,000 data points per dyad. Next, the Displacement (Displ) scores were calculated from the X-Y coordinates. Equation (1) shows how this was done:

$$
\operatorname{Displ}_{t}=\sqrt{\left(\mathrm{X}_{(\mathrm{t}+1)}-\mathrm{X}_{\mathrm{t}}\right)^{2}+\left(\mathrm{Y}_{\mathrm{t}+1}-\mathrm{Y}_{\mathrm{t}}\right)^{2}},
$$

where $\mathrm{X}$ represents the raw medial-lateral measure and $\mathrm{Y}$ the anterior-posterior measure of postural sway.

The Cross Recurrence Quantification Analyses (CRQA) on the Displ data were analyzed in Matlab $^{\circledR}$ (Mathworks Inc., 2012) using the Cross Recurrence Plot (CRP) Toolbox (http://tocsy.pik-potsdam.de; [32]) and casnet [33], a package for the R language [34]. To perform CRQA, the shared phase 

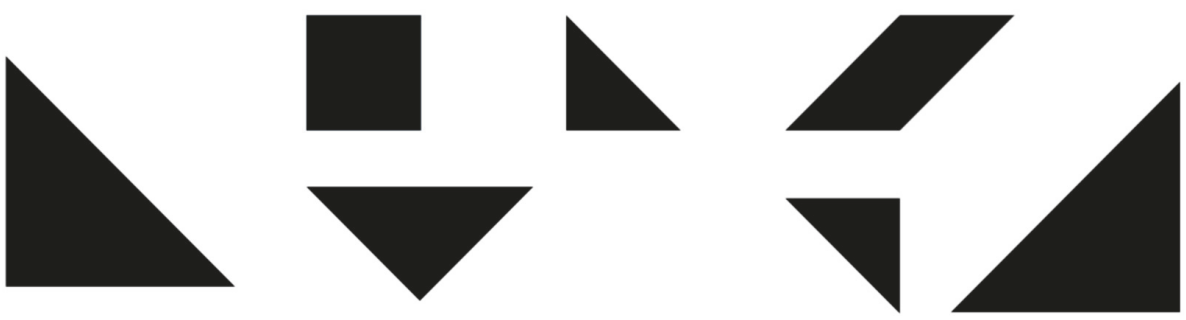

Figure 1: Tangram puzzle pieces.

space of the dyadic time series was reconstructed using the method of time-delayed embedding [35]. To determine an appropriate delay, the Average Mutual Information (AMI) was calculated over increasing time lags. The time lag where the first local minimum (hence, the point where the time series reveal an optimum amount of unique information) appeared was chosen for the reconstruction (5 data points). Next, the embedding dimension (7) was determined by a first local minimum of False Nearest Neighbors (FNN; cf. [36]). The radius (i.e., the area in the shared phase space where revisiting trajectories are considered recurrent) was allowed to vary within each dyad, so that the recurrence rate within each dyad was exactly $5 \%$ (cf. [37]). These parameters were used to optimize the reconstruction. However, as Riley et al. [36] stated, for recurrence analyses on postural sway data, the choices for time lag and embedding dimension are not crucial, but a way to optimize the phase space reconstruction. Before analysis, the time series were rescaled to the maximum phase space diameter [38]

\subsubsection{Descriptive Analysis of Leading-Following Behavior in} Postural Sway. From the CRQA analyses we extracted for each dyad the diagonal-wise recurrence rate (see [39], for a detailed description). For each dyad, a diagonal recurrence profile (DRP) was obtained within a window of 200 samples above and below the LOS (i.e., 40 seconds, $5 \mathrm{~Hz}$ ). A DRP says something about "... how much coordination occurs within a "window" of relative time between participants" and "... the DRP allows us to explore similarities in patterns of movement that are independent of absolute time while revealing patterns of relative time" [40, p. 6]. We chose to look at the determinism measure (DET) within the diagonal profile, which is the percentage of recurring points that lie on a diagonal line in the Cross Recurrence Plot (CRP). This measure tells us something about the nature of the coupling between the two time series as it records recurring shared trajectories that last longer than just 1 point in time. In short, a DRP can tell us something about leading and following behavior in the interaction dynamics of postural sway that evolved during cooperative problem solving.

To make sure that all DRPs are comparable, we chose to place the dyad member that performed best on the pretest on the left side (the y-axis in the Cross Recurrence Plot, see Figure 2) and the dyad member that made less puzzles correct on the right side of the plot (the $\mathrm{x}$-axis in the CRP). DRPs should be interpreted as follows (see also Figures 2 and 3 ). If the determinism peak is left of the middle, the

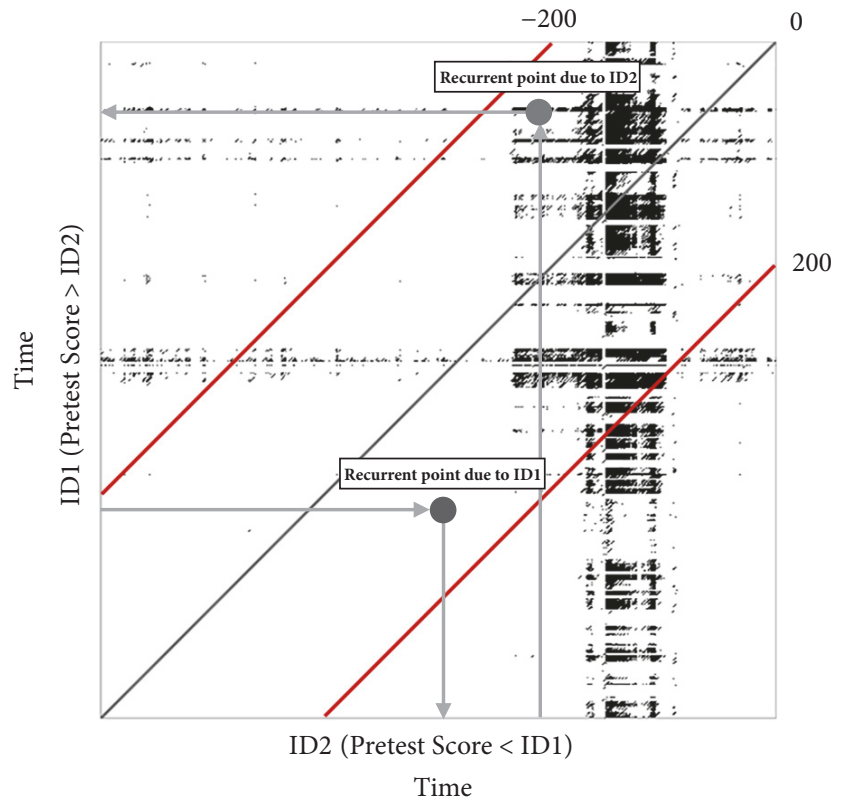

FIgURe 2: A Cross Recurrence Plot of Dyad 47 during the cooperation condition.

worse performing dyad member was in the lead and the better performing dyad member followed. If the determinism peak is right of the middle, the better performing member was in the lead and the worse performing dyad member followed. The distance from the middle to the peak indicates the time lag between the recurrent patterns: the larger the distance is, the longer it took the follower to follow the leader's movement. When the peak is approximately in the middle, there is near-synchronized behavior, indicating that each member did the same thing at about the same time and there was no clear leader or follower. A peak on both sides indicates bi-directionality or turn taking in the interaction. Sometimes the better performing dyad member was in the lead and sometimes the worse performing dyad member was in the lead.

Figure 2 presents a Cross Recurrence Plot of a dyad during cooperation. ID1 on the $y$-axis is the child with a higher pretest score than ID2, who is on the x-axis. Recurrent points below the line of synchrony (LOS, the matrix diagonal) are due to ID1: the recurring value first occurred in the time series of ID1 and later in the time series of ID2. Similarly, recurrent points above the LOS are due to ID2. The DRP 
based on determinism (DET) is constructed by calculating the proportion of recurrent points that form a line for each matrix diagonal contained in the window around the LOS (between the red lines). DET values from diagonals above the LOS are displayed on the left side of the DRP (window range $-200: 0$ in Figures 3 and 4), values from diagonals below the LOS are displayed on the right side in the DRP (window range 0:200 in Figures 3 and 4).

The DRPs of individual dyads were grouped based on their cooperative task performance: those that scored low ( $0 \%-25 \%$ percentile), average (25\%-75\% percentile), and high (75\%-100\% percentile); within these percentile groups the DRPs were aggregated across participants in the regular and special education groups separately. The low-scoring dyads finished 3-6 puzzles, the average group finished 7-10 puzzles, and the high-scoring group finished 11-15 puzzles. Note that for the children attending special-primary education, only five dyads finished 11-15 puzzles.

The aggregated DRPs are shown in Figure 4. These are so-called centroids obtained by using the shape extraction function algorithm in R package dtwclust [41]. The algorithm uses a shape-based distance metric on coefficient-normalized cross-correlation functions to generate the mean shape, or centroid profile from set of different time series (cf. [42]). The spiky grey lines in the upper part of Figure 4 are the extracted mean profile centroids, which were smoothed for clarity of presentation (loess, span $=.2$ ). In addition, the bottom two rows represent the mean score $+95 \%$ bootstrapped CI. We chose to look at DET ( $z$ score, represented on the y-axis), as this measure tells us something about the long-range recurrent trajectories in an interaction and not only about occasional steady point similarities.

2.3.3. Permutation Test of Group Profile Differences. The blue vertical lines in Figure 4 represent significant differences between the regular and special education centroids. A p value for the observed difference can be constructed by conducting a permutation test in which the temporal order of values in each time series is resampled many times, after which difference scores are computed on the resampled series. For each percentile group, the extracted centroids in the DRP were resampled 9999 times using the method of random block size resampling implemented in function tsboot from R package boot (v. 1.3-20, [43]). Because observed time series are autocorrelated, robust resampling is often achieved by defining blocks (bins) that cover the time series and by randomizing those blocks while keeping the sequential order of values within a block as observed [44]. In our permutation analysis block sizes were variable and drawn from a geometric distribution with a mean of 5 (the partial autocorrelation function of the series of observed differences yielded significant correlations up to 4-6 lags). The permutation test evaluates the rank of the observed difference score among the 9999 resampled difference scores for each time point (i.e., ranging from 1 to 10,000). A p value can be calculated by dividing the number of difference scores that are equal to the observed difference or more extreme, on the number of values in the distribution. If the observed value would have had rank 1 , the associated p value would be .0001 . The alpha level was adjusted for multiple comparisons from .05 by a factor of 3 , because the 3 analyses for each percentile group are based on subsets of the independent samples from each school type. The blue lines in Figure 4 correspond to observed differences with a $\mathrm{p}$ value $<.017$.

\section{Results}

Table 1 presents the descriptive statistics of the task performance scores of the individuals and dyads, for both the typically and atypically developing children. The table reveals that dyads performed better than individuals and that typically developing children performed better than children with a neurodevelopmental disorder.

Next, we describe the results of the leader-follower analyses. We chose to use the individual task performance to distinguish between the individuals making up a dyad. Figure 4 shows the leader-follower results of the three performance groups (low, average, and high), distinguishing between typically developing children (i.e., the lighter line) and children with a neurodevelopmental disorder (i.e., the darker line). Below each graph the number of puzzles corrected by the dyad and by each dyad member individually are plotted ( $\mathrm{P} 1$ is the high-performing dyad member; $\mathrm{P} 2$ is the low-performing dyad member). As the scores below the graph show, both groups did not appear to differ a lot on individual scores and cooperative scores.

For low-performing dyads, there was a similar pattern for children attending regular-primary education and children attending special-primary education. For both groups, the best performing child was in the lead, while the child who performed lowest on the individual task was the follower. The peak, however, was far to the right, indicating that it took some time before the worse performing child followed the lead of the better performing child.

For average-performing dyads, the pattern appeared quite similar to that of the low-performing dyads, but only for the children attending regular-primary education. Here, again, the better performing child was in the lead. The peak moved closer to the center, indicating that leading-following took place closer in time than the lowest performing group. For the special education group, however, a different pattern was observed. The peak had moved slightly to the left side of the graph, indicating that the worse performing child was in the lead. In addition, the leading-following pattern took place closely in time, near the line of synchrony. Thus, the postural sway patterns in average-performing dyads of special education children were nearly synchronized.

For high-performing dyads, the results of the groups were opposite. Among children attending regular-primary education, the better performing child was still the leader. The peak had shifted even more towards the LOS, indicating that there was less of a delay between leading and following. Among children with a neurodevelopmental disorder, however, the peak had moved to the left side of the graph, indicating that the best performing dyads were led by the low-performing dyad member. 
ID1 LEADS interaction

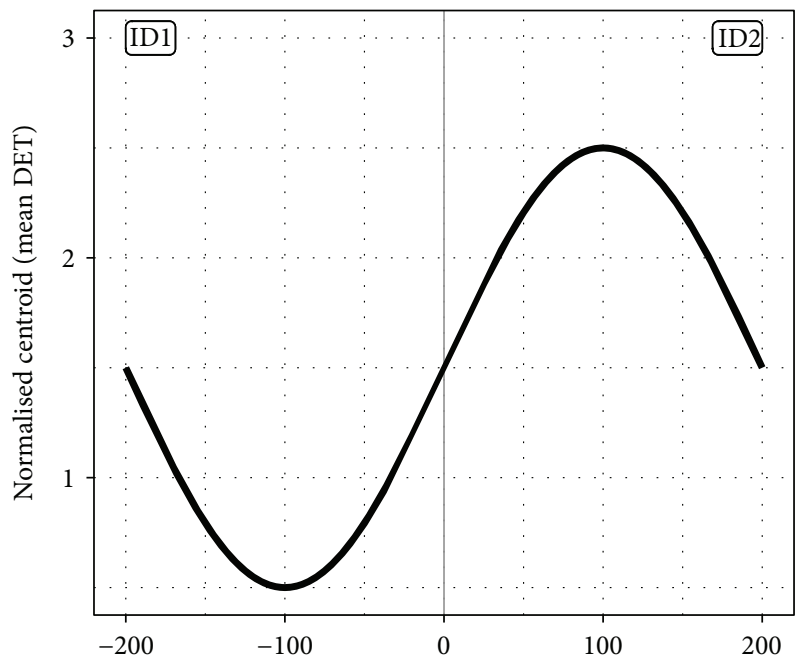

SYNCHRONY

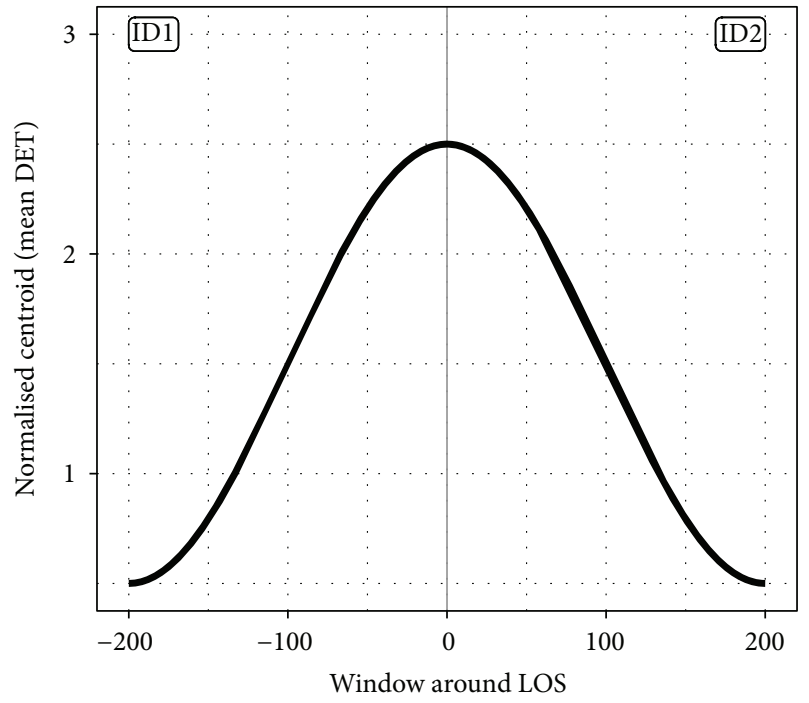

ID2 LEADS interaction

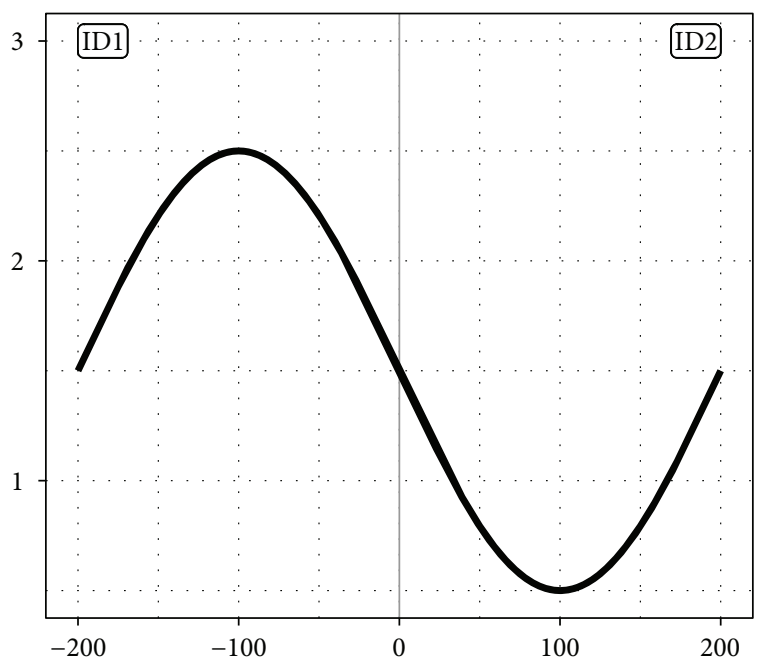

BI-DIRECTIONAL interaction

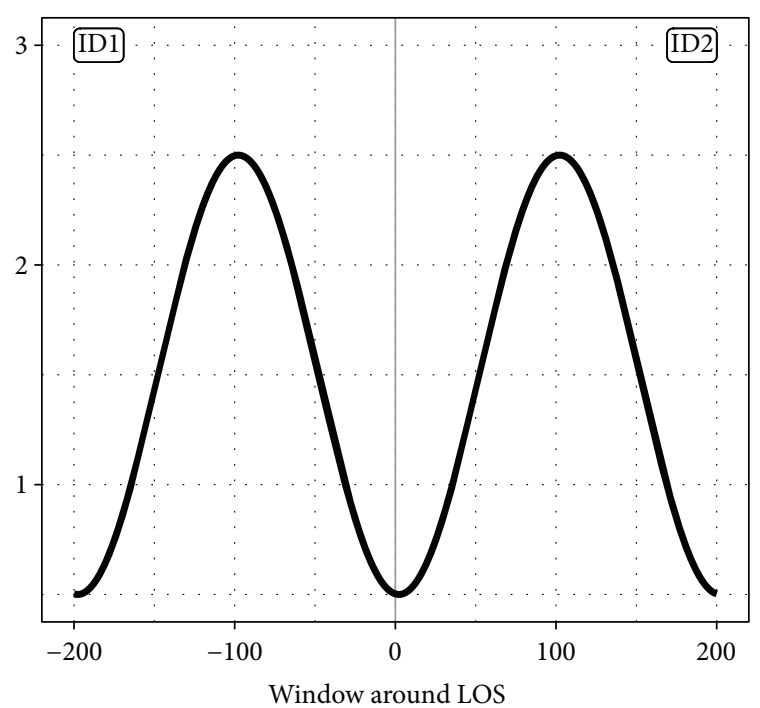

FiguRE 3: Examples of ideal diagonal-wise recurrence profiles showing possible leading-following patterns.

TABLE 1: Descriptive statistics of individual and cooperative task performance for children attending regular and special primary education.

\begin{tabular}{|c|c|c|c|c|c|}
\hline & $N$ & $M$ & $S D$ & Min & $\operatorname{Max}$ \\
\hline \multicolumn{6}{|c|}{ Regular Education } \\
\hline Individually & 366 & 5.73 & 2.29 & 0 & 14 \\
\hline Cooperation & 183 & 9.17 & 2.55 & 5 & 15 \\
\hline \multicolumn{6}{|c|}{ Special Education } \\
\hline Individually & 212 & 3.97 & 1.93 & 0 & 9 \\
\hline Cooperation & 106 & 6.75 & 1.81 & 3 & 13 \\
\hline
\end{tabular}

To summarize, leading-following among children attending regular-primary education remained quite similar in all three ability groups, the better performing dyad member was in the lead. What changed was the fact that across groups, moving from low- to high-performing dyads, there was a decrease in the delay between the leader and follower's postural sway. For children attending special-primary education, there was a clear difference between the three ability groups. In the low-performing dyads it was clearly the better performing dyad member that was in the lead. The exact 

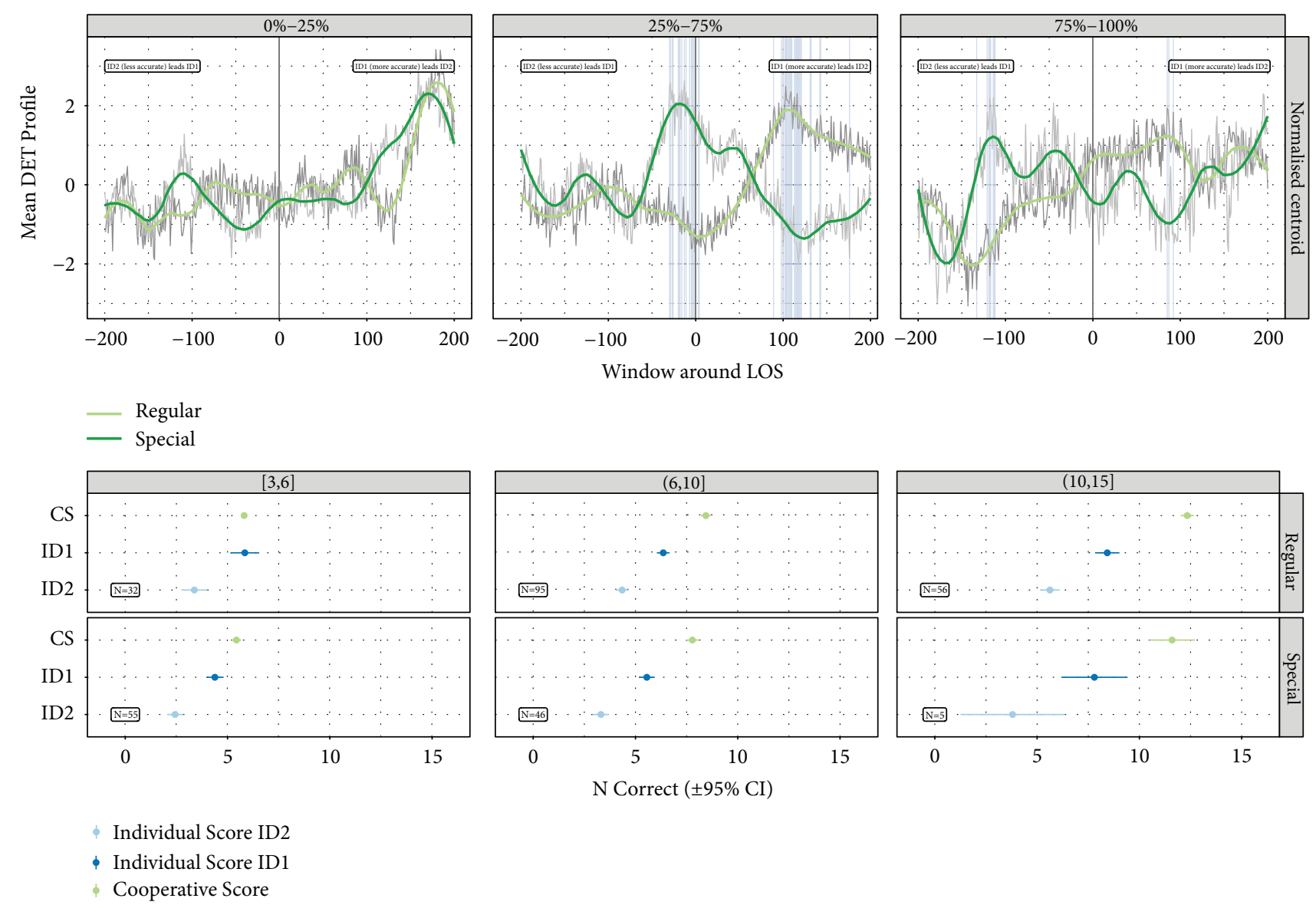

FIGURE 4: The upper panel (1st row) shows the leading-following centroid profiles for regular (light green) and special (dark green) primary education taken from a 40 second window (sampled at $5 \mathrm{~Hz}$ ) around the line of synchronization (LOS) in the CRP. Columns 1-3 represent percentiles of the scores observed in the cooperative condition (lowest $25 \%$, average $50 \%$, and highest $25 \%$, respectively). Prior to averaging, participants were sorted such that the highest scoring individuals on the premeasure in each dyad are on the left side of the LOS. Labels in the upper panel indicating which dyad member would be leading the interaction were a peak observed in that part of the profile. The blue vertical lines indicate at which points the regular and special education centroids differ significantly according to the permutation test (see text for details). The lower panels (2nd and 3rd rows) show the number of dyads and their performance in terms of correct puzzles in each group during cooperation (CS), and premeasure for the high-performing (ID1) and low-performing dyad member (ID2).

opposite was true for the best performing dyads, in which the low-performing dyad member was in the lead. The average group was somewhat in between these two, tending towards a more synchronized interaction.

\section{Discussion}

This study revealed that typically developing children exhibited relatively consistent coordination behavior when cooperating. The more skilled child (i.e., the one with the most correct puzzles on the individual measure) was the leader and the lesser skilled the follower. Note, however, the subtle difference between high- and low-performing dyads: in highperforming dyads, the less skilled child followed the "leader" more closely in time, which was visible in a decrease in delay across performance groups. Thus, the better performing dyads appear to have a more closely matched (in time) pattern of postural sway than low-performing dyads, suggesting that they are more optimally coordinated, which is consistent with findings by Abney et al. [16] and Vink et al. [17, 18].

A possible explanation for the success of typically developing children is that in the high-performing dyads the more cognitively skilled child may have been better at explaining or mediating how the task should be done. Fawcett and Garton [7] showed that it is not only about dyad composition, but that interaction also matters. The nature of the interaction is important considering the zone of proximal development [5]. By stepping into the less skilled child's zone of proximal development, the more skilled child may be able to increase the less skilled child's ability level. In addition, the more skilled child also has to be able to abandon the zone of proximal development of the less skilled child at some point, such that a state of disequilibrium in the interaction can emerge, a prerequisite for learning [45]. By stepping out of the zone of proximal development (i.e., stepping out of the state of equilibrium), the more skilled follower temporarily becomes 
the leader. This way, the less skilled child will experience a state of disequilibrium and may be invited to follow the leader in the hopes of regaining a state of equilibrium.

Another reason for the observed pattern in the typically developing dyads is that interactions will become more and more natural (i.e., more optimally coordinated), with members becoming more equal as skill increases. This is what Leonardi et al. [14] demonstrated in mother-infant vocal interactions. Following behavior by the mother decreased over time (i.e., as the level of competence increased), suggesting that coordination between mother and child increased (i.e., improved), with the interacting individuals becoming more similar or equal over time.

Children with a neurodevelopmental disorder, however, showed a less consistent pattern. In low-performing dyads, the more skilled child was in the lead, whereas in highperforming dyads the less skilled child entrained the more skilled child. In average-performing dyads, there was not a clear leader as indicated by their near-synchronized patterns of postural sway. Thus, typically developing children need a skilled leader, whereas children with a neurodevelopmental disorder in a high-performing dyad need a skilled follower.

As with typically developing children, we can also relate the results of the children with a neurodevelopmental disorder to Vygotsky's [5] zone of proximal development. Unlike typically developing dyads in which the more skilled child could mediate the solution (or process) to the less skilled child, in dyads of children with neurodevelopmental disorders the more skilled child may need to adjust to the needs of the less skilled child. Thus, in this latter group it appears to be important, at least for cognitive performance, that help or mediation is adjusted to the task as well as to children's needs. In other words, the zone of proximal development differs depending on whether one looks at the macro or micro level of behavior, and both are important.

The results of the present study also provide a more detailed picture of the coordination that takes place in the interaction between primary-school children. Abney et al. [16] and Vink et al. [17] showed that level of coordination was related to cooperative cognitive task performance. In addition, Vink et al. [18] showed that this pattern was the same for typically developing children and children with a neurodevelopmental disorder; that is, for dyads consisting of typically developing children as well as those consisting of children with a neurodevelopmental disorder, better task performance was accompanied by more coordinated behavior during the task, as was indicated by less deterministic and more chaotic patterns of interpersonal postural sway. The latter study, however, did not explain how a potential source of information can account for the observed difference in performance between these two groups. In the present study we showed that patterns of leading and following provide one such source of information. Although both groups showed similar leader-follower patterns in the lowest performing group, the patterns were opposite in the best performing dyads.

Our findings have important implications for educational and clinical practice. Teachers should be aware that cooperative learning is strongly influenced by dyad composition.
Cognitive ability differences between dyad members not only determine group performance, but also affect who is leading and who is following. Dyad composition should be adapted to the goal of the task or the goal of one or both of the dyad members. In some cases, this may mean that an educational (or even social) goal of one dyad member conflicts with the goal of the other member. For example, a high-ability student with a neurodevelopmental disorder cooperating with a lowability student may not profit as much from a collaborative task as his or her low-ability peer. Teachers may consider having children collaborate on one occasion with a child who is cognitively superior and on another with a child who is cognitively inferior.

Clinicians may want to learn from our study that children with a neurodevelopmental disorder should sometimes be put in a position in which they are allowed to take the lead. A quote from Gernsbacher [28, p. 145] concludes our message beautifully: "experience suggests that this is when parents-and professionals-need to enact even more reciprocity, need to share even more of the child's world, need to follow even more of the child's lead..."

\section{Data Availability}

Data files and analysis scripts used to produce the results presented here are available from the Open Science Framework (https://osf.io/cfgh7/).

\section{Conflicts of Interest}

The authors declare that they have no conflicts of interest.

\section{Acknowledgments}

Note that the samples described in this study have been used in earlier studies of Vink et al. [17, 18].

\section{References}

[1] R. E. Slavin, "Cooperative learning and academic achievement: Why does groupwork work?" Anales de Psicología, vol. 30, no. 3, pp. 785-791, 2014.

[2] D. W. Johnson and R. T. Johnson, "An educational psychology success story: social interdependence theory and cooperative learning," Educational Researcher, vol. 38, no. 5, pp. 365-379, 2009.

[3] D. W. Johnson, G. Maruyama, R. T. Johnson, D. Nelson, and L. Skon, "Effects of cooperative, competitive, and individualistic goal structures on achievement: a meta-analysis," Psychological Bulletin, vol. 89, no. 1, pp. 47-62, 1981.

[4] C. J. Roseth, D. W. Johnson, and R. T. Johnson, "Promoting Early Adolescents' Achievement and Peer Relationships: The Effects of Cooperative, Competitive, and Individualistic Goal Structures," Psychological Bulletin, vol. 134, no. 2, pp. 223-246, 2008.

[5] L. S. Vygotsky, Mind in society, Harvard University Press, Cambridge, MA, USA, 1978. 
[6] A. F. Garton and C. Pratt, "Peer assistance in childrens problem solving," British Journal of Developmental Psychology, vol. 19, Article ID 026151001166092, pp. 307-318, 2001.

[7] L. M. Fawcett and A. F. Garton, "The effect of peer collaboration on childrens problem-solving ability," British Journal of Educational Psychology, vol. 75, Article ID 000709904, pp. 157-169, 2005.

[8] S. Hooper, "Effects of Peer Interaction During Computer-Based Mathematics Instruction," The Journal of Educational Research, vol. 85, no. 3, pp. 180-189, 1992.

[9] M. Dingel and W. Wei, "Influences on peer evaluation in a group project: An exploration of leadership, demographics and course performance," in Assessment \& Evaluation in Higher Education, vol. 39, pp. 729-742, 2014.

[10] R. L. Dunbar, M. J. Dingel, L. F. Dame, J. Winchip, and A. M. Petzold, "Student social self-efficacy, leadership status, and academic performance in collaborative learning environments," Studies in Higher Education, pp. 10-1080, 2016.

[11] M. Wichers, "The dynamic nature of depression: A new microlevel perspective of mental disorder that meets current challenges," Psychological Medicine, vol. 44, no. 7, pp. 1349-1360, 2014.

[12] A. Chang, S. R. Livingstone, D. J. Bosnyak, and L. J. Trainor, "Body sway reflects leadership in joint music performance," in Proceedings of the National Academy of Sciences, 2017.

[13] M. Guevara, R. F. Cox, M. van Dijk, and P. van Geert, "Attractor dynamics of dyadic interaction: A recurrence based analysis," Nonlinear Dynamics, Psychology, and Life Sciences, vol. 21, pp. 289-317, 2017.

[14] G. Leonardi, I. Nomikou, K. Rohlfing, J. Raczaszek-Leonardi, and J. Rączaszek-Leonardi, "Vocal interactions at the dawn of communication: The emergence of mutuality and complementarity in mother-infant interaction," in Proceedings of the 6th joint IEEE International Conference on Development and Learning and Epigenetic Robotics (ICDL-Epirob), pp. 288-293, Cergy-Pontoise, september 2016.

[15] A. S. Warlaumont, J. A. Richards, J. Gilkerson, and D. K. Oller, "A Social Feedback Loop for Speech Development and Its Reduction in Autism," Psychological Science, vol. 25, no. 7, pp. 1314-1324, 2014.

[16] D. Abney, A. Paxton, R. Dale, and C. T. Kello, "Movement dynamics reflect a functional role for weak coupling and role structure in dyadic problem solving," Cognitive Processing, vol. 16, pp. 325-332, 2015.

[17] R. Vink, M. L. Wijnants, A. H. N. Cillessen, and A. M. T. Bosman, "Cooperative learning and interpersonal synchrony," Nonlinear Dynamics, Psychology, and Life Sciences, vol. 21, no. 2, pp. 189-215, 2017.

[18] R. Vink, F. Hasselman, M. L. Wijnants, A. H. N. Cillessen, and A. M. T. Bosman, Coordinating Postural Sway: Do Children with And without A Neurodevelopmental Disorder Differ?, 2018, https://doi.org/10.31234/osf.io/n96y7.

[19] J. Jaffe, B. Beebe, S. Feldstein et al., "Rhythms of dialogue in infancy: Coordinated timing in development," Monographs of the Society for Research in Child Development, vol. 66, 149 pages, 2001, PMID: 11428150.

[20] E. Lenneberg, Biological Foundations of Language, Wiley, New York, NY, USA, 1967.

[21] B. Beebe, F. Lachman, and J. Jaffe, "Mother-infant interactions structures and presymbolic self- and object representations," Psychoanalytic Dialogues, vol. 7, pp. 133-182, 1997.
[22] B. Beebe, J. Jaffe, F. Lachmann, S. Feldstein, C. Crown, and M. Jasnow, "Systems models in development and psychoanalysis: The case of vocal rhythm coordination and attachment," Infant Mental Health Journal, vol. 21, pp. 99-122, 2000.

[23] R. Mogan, R. Fischer, and J. A. Bulbulia, “To be in synchrony or not? A meta-analysis of synchrony's effects on behavior, perception, cognition and affect," Journal of Experimental Social Psychology, vol. 72, pp. 13-20, 2017.

[24] I. M. Vicaria and L. Dickens, "Meta-analyses of the intra- and interpersonal outcomes of interpersonal coordination," Journal of Nonverbal Behavior, vol. 40, pp. 335-361, 2016.

[25] J. M. Gottman, Marital interactions: Experimental investigations, Academic Press, New York, NY, USA, 1979.

[26] E. Tiegerman and L. H. Primavera, "Imitating the autistic child: Facilitating communicative gaze behavior," Journal of Autism and Developmental Disorders, vol. 14, no. 1, pp. 27-38, 1984.

[27] C. Trevarthen and S. Daniel, "Disorganized rhythm and synchrony: Early signs of autism and Rett syndrome," Brain \& Development, vol. 27, pp. S25-S35, 2005.

[28] M. A. Gernsbacher, "Toward a behavior of reciprocity," Journal of Developmental Processes, vol. 1, Article ID 25598865, pp. 139$152,2006$.

[29] G. Underwood, M. McCaffrey, and J. Underwood, "Gender differences in a cooperative computer-based language task," Educational Research, vol. 32, pp. 44-49, 1990.

[30] R. A. Clark, A. L. Bryant, Y. Pua, P. McCrory, K. Bennell, and M. Hunt, "Validity and reliability of the Nintendo Wii Balance Board for assessment of standing balance," Gait \& Posture, vol. 31, pp. 307-310, 2010.

[31] R. A. Clark, R. McGough, and K. Paterson, "Reliability of an inexpensive and portable dynamic weight bearing asymmetry assessment system incorporating dual Nintendo Wii Balance Boards," Gait \& Posture, vol. 34, no. 2, pp. 288-291, 2011.

[32] N. Marwan, M. C. Romano, M. Thiel, and J. Kurths, "Recurrence plots for the analysis of complex systems," Physics Reports, vol. 438, no. 5-6, pp. 237-329, 2007.

[33] F. Hasselman, "casnet, A toolbox for studying Complex Adaptive Systems and NETworks," https://github.com/FredHasselman/casnet.

[34] R Core Team, A language and environment for statistical computing, Foundation for Statistical Computing, Vienna, Austria, 2017, https://www.R-project.org/.

[35] F. Takens, "Detecting strange attractors in turbulence," in Dynamical systems and Turbulence, D. A. Rand and L. S. Young, Eds., vol. 898 of Lecture Note in Mathematics, pp. 366-381, Springer, Berlin, Germany, 1981.

[36] M. A. Riley, R. Balasubramaniam, and M. T. Turvey, "Recurrence quantification analysis of postural fluctuations," Gait \& Posture, vol. 9, no. 1, pp. 65-78, 1999.

[37] M. L. Wijnants, A. M. T. Bosman, F. Hasselman, R. F. A. Cox, and G. Van Orden, "1/f scaling in movement time changes with practice in precision aiming," Nonlinear Dynamics, Psychology, and Life Sciences, vol. 13, pp. 75-94, 2009.

[38] N. Marwan, "Cross Recurrence Plot Toolbox for MATLAB ${ }^{\circledR}$, Ver. 5.22 (R32.2)," http://tocsy.pik-potsdam.de/CRPtoolbox/.

[39] M. I. Coco and R. Dale, "Cross-recurrence quantification analysis of categorical and continuous time series: An R package," Frontiers in Psychology, vol. 5, p. 14, 2014.

[40] A. Paxton and R. Dale, "Argument disrupts interpersonal synchrony," The Quarterly Journal of Experimental Psychology, vol. 66, no. 11, pp. 2092-2102, 2013. 
[41] A. Sarda-Espinosa, Time Series Clustering Along with Optimizations for the Dynamic Time Warping Distance. R package version 4.0.1, 2017, https://CRAN.R-project.org/package.

[42] J. Paparrizos and L. Gravano, "k-Shape: Efficient and Accurate Clustering of Time Series," in Proceedings of the 2015 ACM SIGMOD International Conference on Management of Data, SIGMOD '15, pp. 1855-1870, 2015.

[43] A. Canty and B. Ripley, "boot, Bootstrap R (S-Plus) Functions. R package version 1.3-20," 2017.

[44] D. N. Politis and J. P. Romano, The stationary bootstrap. of the American Statistical Association, vol. 89, Article ID 2290993, pp. 1303-1313, 1994.

[45] J. Piaget, The language and thought of the child, Routledge and Kegan Paul, London, 3rd edition, 1959. 


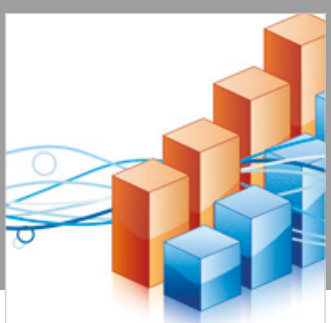

Advances in

Operations Research

\section{-n-m}
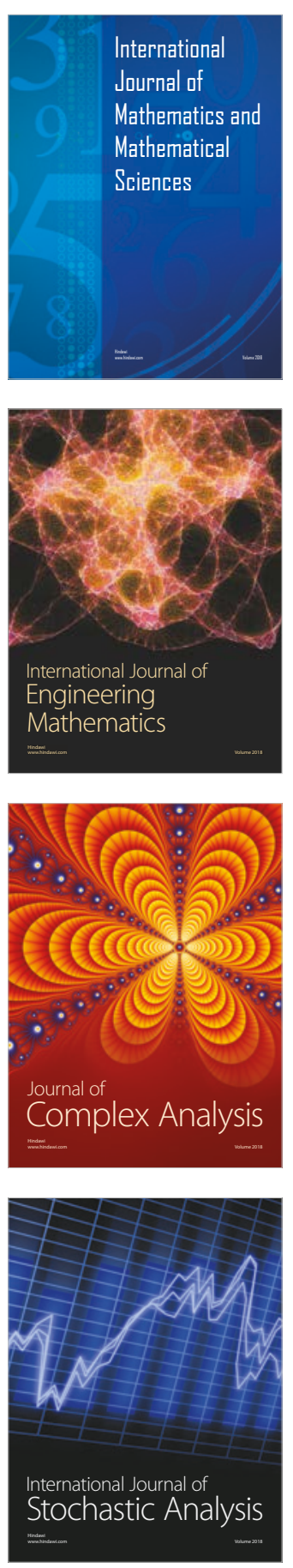
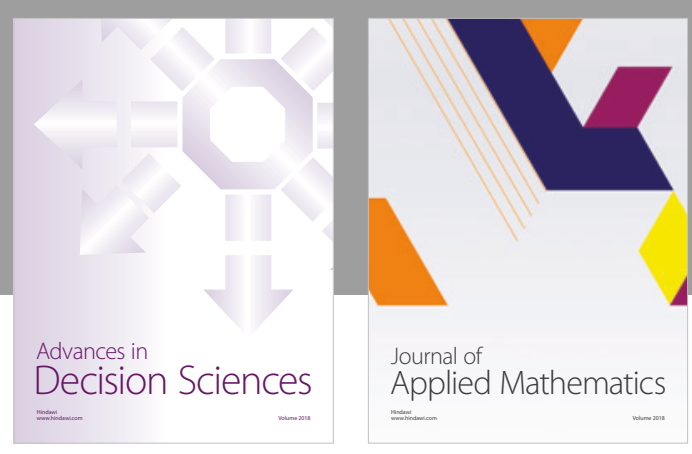

Journal of

Applied Mathematics
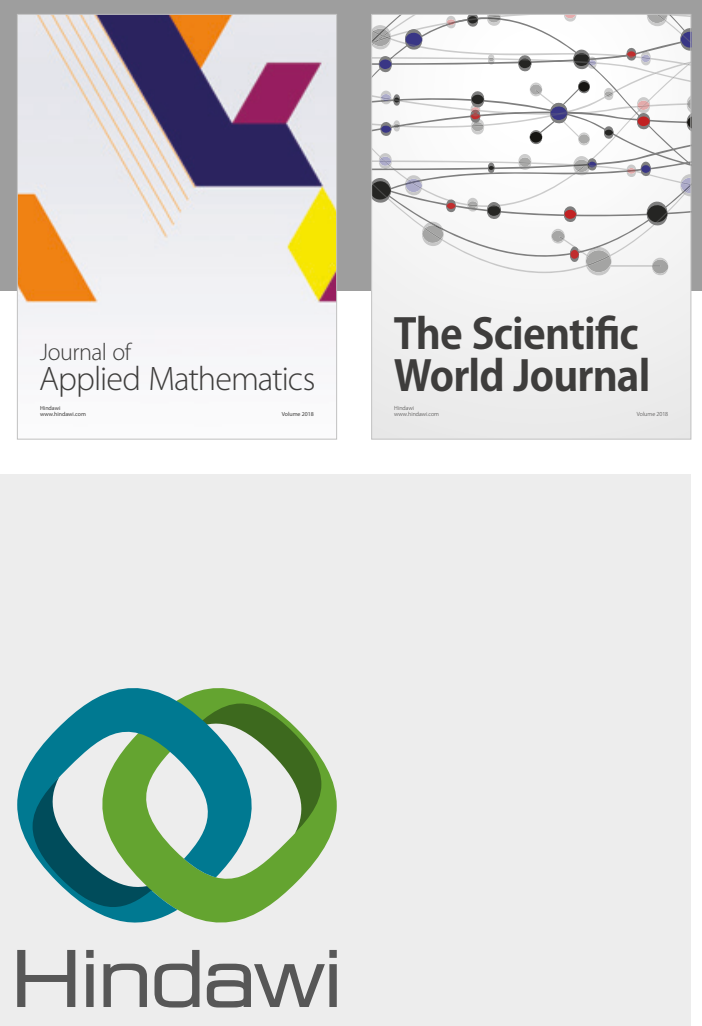

Submit your manuscripts at

www.hindawi.com

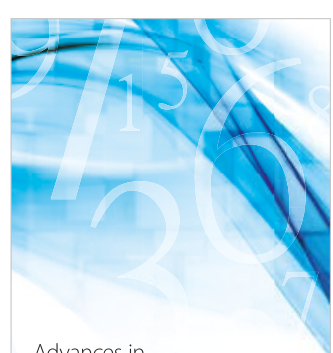

Advances in
Numerical Analysis
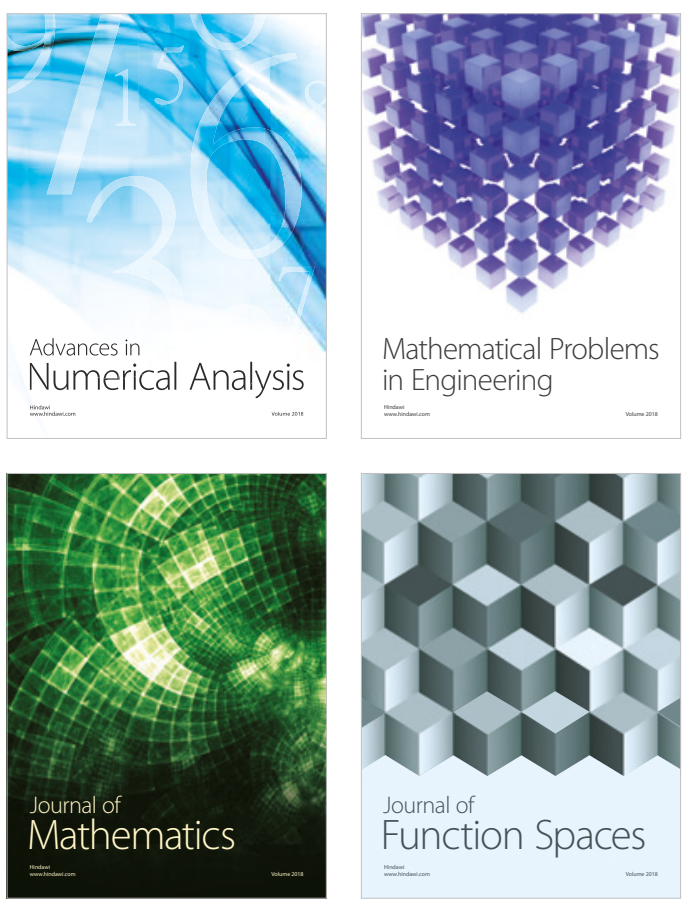

Mathematical Problems in Engineering

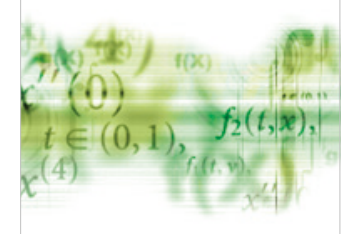

International Journal of

Differential Equations

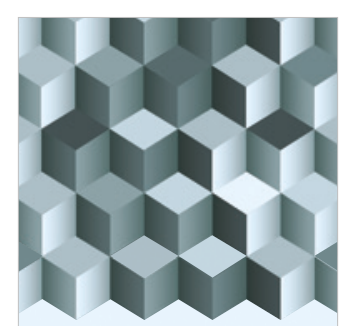

Journal of

Function Spaces
The Scientific

World Journal

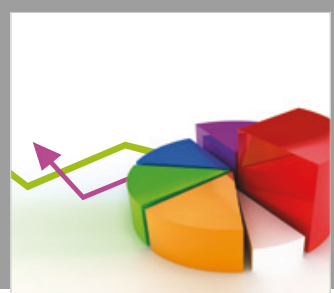

Journal of

Probability and Statistics
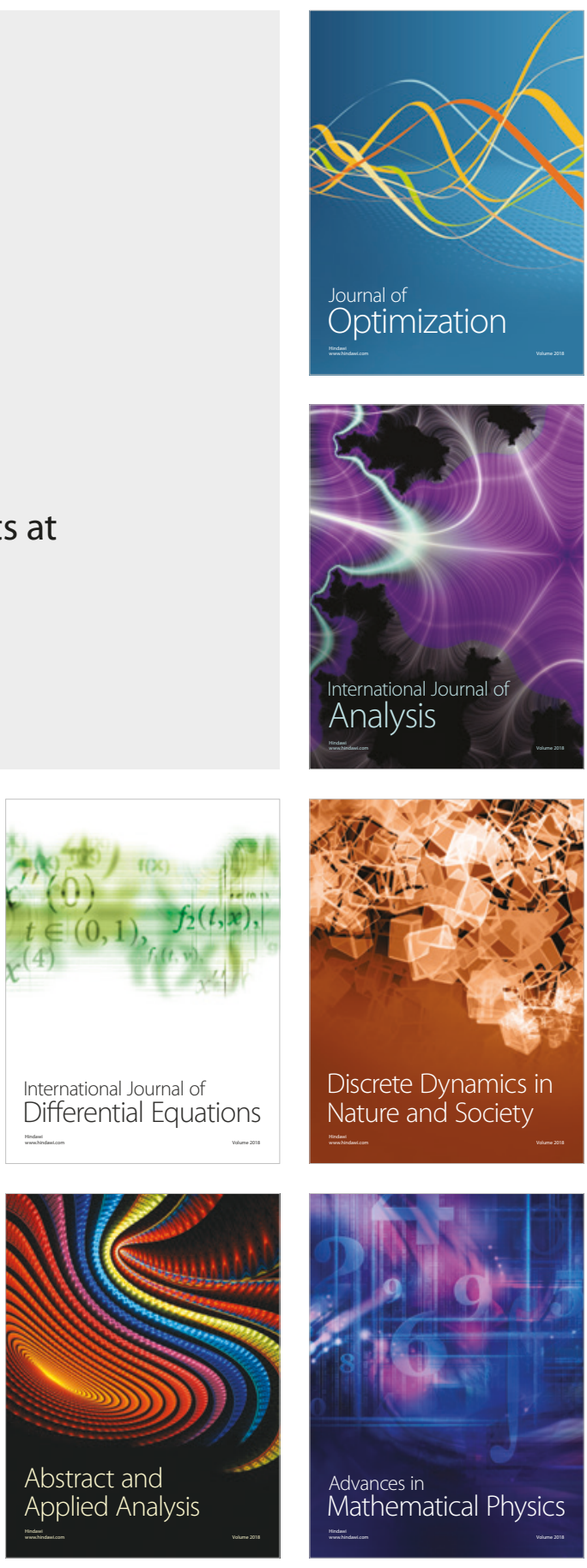